Chapter 8

\title{
Discrete-Time Fractional-Order Systems: Modeling and Stability Issues
}

\author{
Saïd Guermah, Saïd Djennoune and \\ Maâmar Bettayeb \\ Additional information is available at the end of the chapter
}

http://dx.doi.org/10.5772/51983

\section{Introduction}

The concepts of non-integer derivative and integral are the foundation of the fractional calculus [1-4]. Non-integer derivative has become nowadays a precious tool, currently used in the study of the behavior of real systems in diverse fields of science and engineering.

Starting from the sixties, the research in this domain of interest has progressively put to light important concepts associated with formulations using non-integer order derivative. Indeed, non-integer order derivative revealed to be a more adequate tool for the understanding of interesting properties shown by various types of physical phenomena, that is, fractality, recursivity, diffusion and/or relaxation phenomena. [5-14].

This tool has enabled substantial advances in the accuracy of description and analysis of these phenomena, namely in the domain of electrochemistry, electromagnetism and electrical machines, thermal systems and heat conduction, transmission, acoustics, viscoelastic materials and robotics. At its turn, the community of system identification and automatic control early benefited from this tool. The corresponding system representations are of the types of infinite-dimensional or distributed-parameter systems, enriched by diverse approximating non-integer-order models, indifferently called fractional-order models. There are abundant successful examples in the literature and one can see some for example in [15-30].

An important feature of fractional-order systems (FOS) is that they exhibit hereditarily properties and long memory transients. This aspect is taken into account in modeling, namely with state-space representation, in parameter estimation, identification, and controller design. Besides, these issues can be viewed either in the scope of a continuous time, or a discrete time representation. In this Chapter, we focus on some issues of modeling and stability of LTI FOS in discrete time. The extension of the results obtained with continuous-time representations to the discrete-time case is evidently motivated by 
the general trend to use digital computer-aided simulations and implementations in control engineering and signal processing. In the particular case of FOS, the discretization methods lead to new models and enlarge the possibilities of representation and simulation of such systems.

More specifically, the familiar state-space representation and its well-established results in the integer-order case receive here an extension to the non-integer case. This extension induces noticeable changes: there appears a neccessity of interpretation of the initial conditions [31] and a neccesity to take into account the history (or memory) of the system from the initial instant, since we have to deal, instead of the classical state, with a so-called "'pseudo state"', that is an expanded state $[32,33]$. This infinite-dimensional system, as we expose it in the following sections, has been studied, from the point of view of its structural properties, i.e., controllability, observability [34,35] and, to some extent, of its stability [36].

\section{LTI continuous-time fractional-order modeling}

In the beginning, most of the works consecrated to the study of FOS focused on continuous-time representations. The continuous-time state-space FOS representation was introduced in [24, 37-40]. It has been employed in analysis of system performances. The solution of the state-space equation was derived by using the Mittag-Lefller function [41]. Next, the stability of FOS was investigated [42] and a condition based on the argument principle was established to guarantee the asymptotic stability of the fractional-order system. Further, the controllability and the observability properties have been defined and some algebraic criteria of these two properties have been derived in [43]. Another contribution to the analysis of the controllability and the observability with commensurate FOS modeled by fractional state-space equations is brought in [44].

\subsection{Definitions of non-integer derivatives}

There are different definitions of the non-integer derivatives [1-3, 45]. Let us consider a function $f(t)$ of the real variable $t$, continuous and integrable on $[a,+\infty[$, where $a$ is the origin of $t$. Indeed, we usually deal with dynamic systems and $f(t)$ is let to be a causal function of $t$, with $t \geq a$. Thus $f(t)=0$ if $t<a$.

Let us introduce the positive integer number $m$ such that $(m-1)<\alpha<m$. We obtain the definition due to A.V. Letnikov

$$
{ }_{a}^{L} D_{t}^{\alpha} f(t)=\frac{1}{\Gamma(m-\alpha+1)} \int_{a}^{t}(t-\tau)^{m-\alpha} f^{(m+1)}(\tau) d \tau+\sum_{k=0}^{m} \frac{f^{(k)}(a)(t-a)^{k-\alpha}}{\Gamma(k-\alpha+1)}, \text { for } t<a .
$$

The definition given by (1) assumes that function $f$ is sufficiently differentiable and that $f^{(k)}(a)<\infty, k=0,1, \ldots, m$. $\Gamma$ is the Euler gamma function

$$
\Gamma(\beta)=\int_{0}^{\infty} z^{\beta-1} e^{-z} d z
$$

in which $z$ is a complex variable. A new version is the Letnikov-Riemann-Liouville definition (LRL) given by 


$$
{ }_{a}^{L} D_{t}^{\alpha} f(t)=\frac{d^{m}}{d t^{m}}\left\{\frac{1}{\Gamma(m-\alpha)} \int_{a}^{t} \frac{f(\tau)}{(t-\tau)^{\alpha-m+1}} d \tau\right\}
$$

Naturally, as physical systems are modeled by differential equations containing eventually fractional derivatives, it is necessary to give to these equations initial conditions that must be physically interpretable. Unfortunately, the LRL definition leads to initial conditions containing the value of the fractional derivative at the initial conditions. To overcome this difficulty, Caputo proposed another definition given by

$$
{ }_{a}^{C} D_{t}^{\alpha} f(t)=\frac{1}{\Gamma(m-\alpha)} \int_{a}^{t} \frac{f^{(m)}(\tau)}{(t-\tau)^{\alpha-m+1}} d \tau
$$

Note the remarkable fact that the LRL fractional derivative of a constant function $f(t)=C$ is not zero

$$
{ }_{a}^{L} D_{t}^{\alpha} C=\frac{C t^{-\alpha}}{\Gamma(1-\alpha)}
$$

whereas Caputo's fractional derivative of a constant is identically zero.

\subsection{FOS differential equation}

Let us now consider a SISO LTI FOS. By means of its dynamic input-output relation and using (4), we can derive its continuous-time models. In all what follows, we use Caputo's definition of a fractional derivative with initial time $t=0$, i.e., $a=0$. The derived differential equation is then expressed by [45]

$$
\sum_{i=1}^{n_{a}} a_{i} D^{\alpha_{i}} y(t)=\sum_{j=1}^{n_{b}} b_{j} D^{\beta_{j}} u(t)
$$

where $a_{i}, b_{j} \in \mathbb{R}, \alpha_{i}, \beta_{j} \in \mathbb{R}^{+}, u(t) \in \mathbb{R}$ is the input of the system and $y(t) \in \mathbb{R}$ its output. $n_{a}$ and $n_{b} \in \mathbb{N}$ are the number of terms of each side of the differential equation. The differential equation is said with commensurate order if all the differentiation orders $\alpha_{i}, \beta_{j}$ are multiple integers of a same base order $\alpha$. In this case, it can be expressed by

$$
\sum_{i=1}^{n_{a}} a_{i} D^{i \alpha} y(t)=\sum_{j=1}^{n_{b}} b_{j} D^{j \alpha} u(t)
$$

\subsection{State-space model derived from a non-integer-order transfer function}

By using Laplace transform, assuming null initial conditions, from (6) we derive the following transfer function

$$
G(s)=\frac{Y(s)}{U(s)}=\frac{\sum_{j=0}^{n_{b}} b_{j} s^{\beta_{j}}}{\sum_{i=0}^{n_{a}} a_{i} s^{\alpha_{i}}}
$$


To facilitate the development of the so-called construction procedure, (8) is rewritten with coefficients $a_{i}$ and $b_{j}$ denoted differently by $a_{2 k+1}^{\prime}$ and $a_{2 k}^{\prime}$ respectively [35]. This gives

$$
G(s)=\frac{Y(s)}{U(s)}=\frac{a_{0}^{\prime}+a_{2}^{\prime} s^{\alpha_{2}}+a_{4}^{\prime} s^{\alpha_{4}}+\ldots+a_{2 n}^{\prime} s^{\alpha_{2 n}}}{1+a_{1}^{\prime} s^{\alpha_{1}}+a_{3}^{\prime} s^{\alpha_{3}}+\ldots+a_{2 n+1}^{\prime} s^{\alpha_{2 n+1}}},
$$

in which $\alpha_{i}$ are the fractional orders that can be either commensurate or non-commensurate, with

$$
\alpha_{1} \leq \alpha_{2} \leq \alpha_{3} \leq \ldots \leq \alpha_{2 n} \leq \alpha_{2 n+1}
$$

The procedure for obtaining a state-space representation from (9) is as follows: firstly, let us introduce an intermediate variable $\mathcal{X}(s)$ such that

$$
G(s)=\frac{Y(s)}{\mathcal{X}(s)} \frac{\mathcal{X}(s)}{U(s)}
$$

Next, we can write

$$
G(s)=\frac{Y(s)}{\mathcal{X}(s)} \frac{\mathcal{X}(s)}{U(s)}=\frac{\left(a_{0}^{\prime}+a_{2}^{\prime} s^{\alpha_{2}}+a_{4}^{\prime} s^{\alpha_{4}}+\ldots+a_{2 n}^{\prime} s^{\alpha_{2 n}}\right) \mathcal{X}(s)}{\left(1+a_{1}^{\prime} s^{\alpha_{1}}+a_{3}^{\prime} s^{\alpha_{3}}+\ldots+a_{2 n+1}^{\prime} s^{\alpha_{2 n+1}}\right) \mathcal{X}(s)}
$$

Let us put successively

$$
\begin{aligned}
\mathcal{X}_{1}(s) & =\mathcal{X}(s) \\
\mathcal{X}_{2}(s) & =s^{\alpha_{1}} \mathcal{X}(s)=s^{\alpha_{1}} \mathcal{X}_{1}(s) \\
\mathcal{X}_{3}(s) & =s^{\alpha_{2}} \mathcal{X}(s)=s^{\left(\alpha_{2}-\alpha_{1}\right)} \mathcal{X}_{2}(s) \\
& \vdots \\
\mathcal{X}_{2 n+1}(s) & =s^{\alpha_{2 n}} \mathcal{X}(s)=s^{\left(\alpha_{2 n}-\alpha_{2 n-1}\right)} \mathcal{X}_{2 n}(s) .
\end{aligned}
$$

With (10) and relations (11), it is easy to build the following group of equations

$$
\begin{aligned}
s^{\alpha_{1}} \mathcal{X}_{1}(s) & =\mathcal{X}_{2}(s) \\
s^{\left(\alpha_{2}-\alpha_{1}\right)} \mathcal{X}_{2}(s) & =\mathcal{X}_{3}(s) \\
\vdots & \\
s^{\left(\alpha_{i}-\alpha_{i-1}\right)} \mathcal{X}_{i}(s) & =\mathcal{X}_{i+1}(s) \\
\vdots & \\
s^{\left(\alpha_{2 n+1}-\alpha_{2 n}\right)} \mathcal{X}_{2 n+1}(s) & =\frac{1}{a_{2 n+1}^{\prime}}\left[U(s)-\mathcal{X}_{1}(s)-a_{1}^{\prime} \mathcal{X}_{2}(s)-\ldots-a_{2 n-1}^{\prime} \mathcal{X}_{2 n}(s)\right]
\end{aligned}
$$


The transposition in the time domain yields a state-space representation of System (9), with $x_{i}(t), u(t)$ and $y(t)$ the respective inverse Laplace transforms of $\mathcal{X}_{i}(s), U(s)$ and $Y(s)$

$$
\begin{array}{clc}
D^{\alpha_{1}} x_{1}(t) & = & x_{2} \\
D^{\left(\alpha_{2}-\alpha_{1}\right)} x_{2}(t) & = & x_{3} \\
\vdots & & \\
D^{\left(\alpha_{i}-\alpha_{i-1}\right)} x_{i}(t) & = & \\
\vdots & & \\
D^{\left(\alpha_{2 n}-\alpha_{2 n-1}\right)} x_{2 n}(t) & = & \\
D^{\left(\alpha_{2 n+1}-\alpha_{2 n}\right)} x_{2 n+1}(t) & =\frac{1}{a_{2 n+1}^{\prime}}\left[u(t)-x_{1}(t)-a_{1}^{\prime} x_{2}(t)-\ldots-a_{2 n-1}^{\prime} x_{2 n}(t)\right],
\end{array}
$$

the expression of the system output being

$$
y(t)=a_{0}^{\prime} x_{1}(t)+0 x_{2}(t)+a_{2}^{\prime} x_{3}(t)+\ldots+0 x_{2 n}+a_{2 n}^{\prime} x_{2 n+1}(t) .
$$

The corresponding state-space group of equations is then derived

$$
D^{[\gamma]} x(t)=A x(t)+B u(t) \quad x(0)=x_{0},
$$

where $x(t)=\left[\begin{array}{lll}x_{1}(t) & x_{2}(t) \ldots & x_{2 n+1}(t)\end{array}\right]^{T} \in \mathbb{R}^{2 n+1}$ is the column state vector and $x(0)$ its initial value. Note that here $x(0)$ can be assumed non null, instead of the assumption of null initial conditions necessary to define the transfer function model. Besides, we have

$$
D^{[\gamma]} x(t)=\left[\begin{array}{c}
D^{\gamma_{1}} x_{1}(t) \\
\vdots \\
D^{\gamma_{2 n+1}} x_{2 n+1}(t)
\end{array}\right]
$$

in which $\gamma_{i} \in \mathbb{R}^{\star+}, \gamma_{1}=\alpha_{1}$ for $i=1$ and $\gamma_{i}=\left(\alpha_{i}-\alpha_{i-1}\right)$ for $i=2, \ldots, 2 n+1$.

The output equation takes the form

$$
y(t)=C x(t)+D u(t)
$$

Matrices A, B, C and D are given by

$$
\begin{aligned}
& A=\left[\begin{array}{ccccccc}
0 & 1 & 0 & 0 & 0 & \ldots & 0 \\
0 & 0 & 1 & 0 & \ldots & \ldots & 0 \\
\vdots & \vdots & \vdots & \vdots & \vdots & \vdots & \vdots \\
0 & 0 & 0 & 0 & \ldots & \ldots & 1 \\
-\frac{1}{a_{2 n+1}^{\prime}} & \frac{-a_{1}^{\prime}}{a_{2 n+1}^{\prime}} & 0 & \frac{-a_{3}^{\prime}}{a_{2 n+1}^{\prime}} & 0 & \ldots & \frac{-a_{2 n-1}^{\prime}}{a_{2 n+1}^{\prime}}
\end{array}\right], \quad B=\left[\begin{array}{c}
0 \\
0 \\
\vdots \\
\frac{1}{a_{2 n+1}^{\prime}}
\end{array}\right] \\
& C=\left[\begin{array}{lllllll}
a_{0}^{\prime} & 0 & a_{2}^{\prime} & 0 & \ldots & 0 & a_{2 n}^{\prime}
\end{array}\right] .
\end{aligned}
$$


As for direct transmission matrix $D$, we have

$$
D=\left[\frac{a_{2 n}^{\prime}}{a_{2 n+1}^{\prime}}\right] \text { if } \alpha_{2 n}=\alpha_{2 n+1} \text {, otherwise } D=[0] \text {. }
$$

The symbol $D$ here should not be confused with the derivation operator $D^{[\cdot]}$.

Remark 1. The dimension of the model, i.e., its number of state variables $2 n+1$ is equal to the total number of non-null terms $a_{l}^{\prime} s^{\alpha_{l}}$ present in the numerator and denominator of $G(s)$. In the following, $2 n+1$ is denoted $n_{d}$.

The controllability and observability properties as well as the stability have been well studied when the differentiation fractional-orders $\gamma_{i}$ are all equal to a unique value, say $\alpha$ ([43], [42]). In this case, (15) becomes

$$
D^{[\alpha]} x(t)=\left[\begin{array}{c}
D^{\alpha} x_{1} \\
D^{\alpha} x_{2} \\
\vdots \\
D^{\alpha} x_{n_{d}}
\end{array}\right]=A x(t)+B u(t), \quad x(0)=x_{0}
$$

Applying the Laplace transform to (17), we have

$$
X(s)=\left[s^{\alpha} \mathbb{I}-A\right]^{-1}[B U(s)+x(0)],
$$

Defining $\Phi(t)=\mathcal{L}^{-1}\left[s^{\alpha} \mathbb{I}-A\right]^{-1}$ as the corresponding state transition matrix, we obtain the state response given by

$$
x(t)=\Phi(t) x(0)+\int_{0}^{t} \Phi(t-\tau) B u(\tau) d \tau
$$

It can be shown that $\Phi(t)$ can be expressed by

$$
\Phi(t)=\sum_{k=0}^{\infty} \frac{A^{k} t^{k \alpha}}{\Gamma(1+k \alpha)}
$$

The left side term of this latter equation represents the Mittag-Leffler function [41] :

$$
E_{\alpha}(t) \triangleq \sum_{k=0}^{\infty} \frac{A^{k} t^{k \alpha}}{\Gamma(1+k \alpha)}
$$

It has been established in [43] that the controllability and observability conditions of the continuous-time state-space representation of commensurate fractional-order systems are the same as in the integer-order case. Thus, System (17) is controllable if the rank of the controllability matrix

$$
\mathcal{C}=\left[\begin{array}{llll}
B & A B & A^{2} B & \ldots A^{n_{d}-1} B
\end{array}\right]
$$


is equal to $n_{d}$. Besides, this system is observable if the rank of the observability matrix

$$
\mathcal{O}=\left[\begin{array}{c}
C \\
C A \\
C A^{2} \\
\vdots \\
C A^{n_{d}-1}
\end{array}\right]
$$

is equal to $n_{d}$. To our knowledge, no such results have been brought in the literature up to now for FOS with non commensurate fractional-order systems in continuous time. The next section of this chapter constitutes a contribution to the study of structural properties of non-commensurate FOS, using a different approach, that is discrete-time representation [34].

\section{LTI discrete-time fractional-order modeling}

The development of a discrete-time model is based on the Grünwald-Letnikov's definition of fractional-order operators. This definition is another expression of (1), based on the generalization of the backward difference. The generalization of the integer-order difference to a non-integer-order (or fractional-order) difference is addressed in [33, 47].

\subsection{The discrete-time fractional-order difference operator}

In what follows, we present a discretized representation of the continuous fractional-order state-space model of Equations (15) and (16), in which the fractional order can be commensurate or non-commensurate.

Let us consider the state-space model with integer-order derivatives

$$
D^{1} x(t)=A x(t)+B u(t)
$$

where $x(t)=\left[\begin{array}{lll}x_{1}(t) & x_{2}(t) \ldots & x_{n_{d}}(t)\end{array}\right]^{T} \in \mathbb{R}^{n_{d}}$ is the state vector. This latter model can be represented in discrete-time using a forward difference approximation of the derivative of order one of $x(t)[32,33]$. For this purpose let us consider a sampling period $h$. Then for $k h \leq t<(k+1) h$ the integer order first derivative $D^{1} x(t)$ can be approximated by

$$
D^{1} x(t) \approx \Delta^{1} x((k+1) h)=\frac{x((k+1) h)-x(k h)}{h} .
$$

We can then write

$$
\Delta^{1}(x(k+1) h)=A x(k h)+B u(k h)
$$

We start with applying a discretization of (17) with identical fractional orders. The fractional derivatives $D^{[\alpha]} x(t)$ can be approximated by using the definition due to K. Grünwald

$$
\left.D^{[\alpha]} x(t) \approx \Delta_{h}^{[\alpha]} x((k+1) h)=\frac{1}{h^{\alpha}} \sum_{j=0}^{k+1}(-1)^{j}\left(\begin{array}{c}
\alpha \\
j
\end{array}\right) x(k+1-j) h\right)
$$


In this equation, $\alpha \in \mathbb{R}^{\star+}$ is the fractional order, $t$ is the current time, $h \in \mathbb{R}^{\star+}$ is the sampling period or time increment. The term $\left(\begin{array}{l}\alpha \\ j\end{array}\right)$ is calculated by the relation

$$
\left(\begin{array}{l}
\alpha \\
j
\end{array}\right)= \begin{cases}1 & \text { for } j=0 \\
\frac{\alpha(\alpha-1) \ldots(\alpha-j+1)}{j !} & \text { for } j>0\end{cases}
$$

If we drop $h$ (i.e., making $h=1$ ), we find the discrete fractional-order difference operator $\Delta^{\alpha}$ as defined and used in $[32,33,46]$. Indeed, the choice of the sampling period $h$ is very important when elaborating the discretized model of a given process. $h$ is not necessarily small. It corresponds to an appropriate sampling rate, deduced from the dynamics (or frequency bandwidth) of the process, by using rules of thumbs and criteria, as exposed for example in [48, 49].

\subsection{The discrete-time fractional-order state-space model}

The previous results conduct to the linear discrete-time fractional-order state-space model

$$
\Delta^{[\alpha]} x(k+1)=A x(k)+B u(k) ; \quad x(0)=x_{0}
$$

In this model, the differentiation order $\alpha$ is taken the same for all the state variables $x_{i}(k)$, $i=1, \ldots, n_{d}$. Besides, from (27) we have

$$
h^{\alpha} \Delta^{[\alpha]} x(k+1)=x(k+1)+\sum_{j=1}^{k+1}(-1)^{j}\left(\begin{array}{c}
\alpha \\
j
\end{array}\right) x(k-j+1)
$$

Substituting (30) into (29) yields

$$
x(k+1)=\tilde{A} x(k)-\sum_{j=1}^{k+1}(-1)^{j}\left(\begin{array}{l}
\alpha \\
j
\end{array}\right) x(k-j+1)+\tilde{B} u(k)
$$

with $\tilde{A}=h^{\alpha} A$ and $\tilde{B}=h^{\alpha} B$. By setting $c_{j}=(-1)^{j}\left(\begin{array}{c}\alpha \\ j\end{array}\right)$, (31) can be rewritten as follows

$$
x(k+1)=\left(\tilde{A}-c_{1} I_{n_{d}}\right) x(k)-\sum_{j=2}^{k+1} c_{j} x(k-j+1)+\tilde{B} u(k)
$$

Let us put now

$$
A_{0}=\left(\tilde{A}-c_{1} I_{n_{d}}\right)
$$

and, for all $j>0$ :

$$
A_{j}=-c_{j+1} I_{n_{d}}
$$


This leads to

$$
x(k+1)=A_{0} x(k)+A_{1} x(k-1)+A_{2} x(k-2)+\ldots+A_{k} x(0)+\tilde{B} u(k)
$$

This description can be extended to FOS with different (commensurate or non-commensurate) orders [53]

$$
\begin{gathered}
\Delta_{h}^{[\gamma]} x(k+1)=A x(k)+B u(k) \\
x(k+1)=\Delta_{h}^{[\gamma]} x(k+1)+\sum_{j=1}^{k+1} A_{j} x(k-j+1)
\end{gathered}
$$

where

$$
\Delta_{h}^{[\gamma]} x(k+1)=\left[\begin{array}{c}
h^{\gamma_{1}} \Delta^{\gamma_{1}} x_{1}(k+1) \\
\vdots \\
h^{\gamma_{n_{d}} \Delta^{\gamma_{n_{d}}} x_{n_{d}}(k+1)}
\end{array}\right]
$$

in which $\gamma_{i} \in \mathbb{R}^{\star+}, i=1,2, \ldots$ denote any fractional orders. Here, we can write

$$
\begin{gathered}
A_{0}=\tilde{A}+\operatorname{diag}\left\{\left(\begin{array}{c}
\gamma_{i} \\
1
\end{array}\right), i=1, \ldots, n_{d}\right. \\
A_{j}=\operatorname{diag}\left\{-(-1)^{j+1}\left(\begin{array}{c}
\gamma_{i} \\
j+1
\end{array}\right), i=1, \ldots, n_{d}\right\}, j=1,2, \ldots
\end{gathered}
$$

Using (35), we obtain the state equation

$$
x(k+1)=\sum_{j=0}^{k} A_{j} x(k-j)+\tilde{B} u(k) ; \quad x(0)=x_{0}
$$

In this case, $\tilde{A}$ and $\tilde{B}$ are calculated as follows $\tilde{A}_{i}=h^{\gamma_{i}} A_{i} ; \tilde{B}_{i}=h^{\gamma_{i}} B_{i}$ where $A_{i}$ and $B_{i}$ denote the rows of $A$ and $B$ respectively. The corresponding output equation is

$$
y(k)=C x(k)
$$

In this resulting discrete time state-space model, $A_{j}$ is given by (32) and (33), in the case of a unique fractional-order while it is given by (35) and (36) in the case of different, commensurate or non-commensurate fractional-orders.

Remark 2. The model defined by (37) and (38) can be viewed as a discrete-time model with time-delay in state. It has a varying number of steps of time-delays, equal to $k$, i.e., increasing with time. Instead, the models addressed in [50-52] consider a finite constant number of steps of time-delays. 
Define $G_{k}$ such that

$$
G_{k}=\left\{\begin{array}{lc}
I_{n_{d}} & \text { for } k=0, \\
\sum_{j=0}^{k-1} A_{j} G_{k-1-j} & \text { for } k \geq 1
\end{array}\right.
$$

Theorem 1. The solution of (37) is given by

$$
x(k)=\mathrm{G}_{k} x(0)+\sum_{j=0}^{k-1} \mathrm{G}_{k-1-j} B u(j)
$$

This theorem can be proved by induction [34]. The first part of the solution of (40) represents the free response of the system and the last part takes the role of the convolution sum corresponding to the forced response.

The corresponding transition matrix can be defined as

$$
\Phi(k, 0)=G_{k}, \quad \Phi(0,0)=G_{0}=I_{n_{d}}
$$

Remark 3. - $\Phi(k, 0)$ exhibits the particularity of being time-varying, in the sense that it is composed of a number of terms $\mathrm{A}_{j}$ which grows along with $k$. This is due to the fractional-order feature of the model which takes into account all the past values of the states.

By virtue of Equations (37) and (38), the discretization of this state-space realization is

$$
\begin{gathered}
x(k+1)=\sum_{j=0}^{k} A_{j} x(k-j)+B u(k) ; \quad x(0)=x_{0} \\
y(k)=C x(k)
\end{gathered}
$$

Here we have

$$
\begin{gathered}
A_{0}=A+\operatorname{diag}\left\{\left(\begin{array}{c}
\alpha_{i} \\
1
\end{array}\right), i=1, \ldots, n_{d}\right\} \\
A_{j}=\operatorname{diag}\left\{-(-1)^{j+1}\left(\begin{array}{c}
\alpha_{i} \\
j+1
\end{array}\right), i=1, \ldots, n_{d}\right\}, j=1,2, \ldots
\end{gathered}
$$

The output response for a given input sequence and initial conditions is given by

$$
y(k)=C G_{k} x(0)+\sum_{j=0}^{k-1} C G_{k-1-j} B u(j)
$$




\section{Structural properties of LTI discrete-time FOS}

\subsection{Reachability and controllability}

We discuss here a fundamental question for dynamic systems modeled by (37) in the case of a non-commensurate fractional-order. This question is to determine whether it is possible to transfer the state of the system from a given initial state to any other state. We search below to extend two concepts of state reachability (or controllability from the origin) and controllability (or controllability to the origin) to the present case. We are interested in completely state reachable and controllable systems.

Definition 1. The linear discrete-time fractional-order system modeled by (37) is reachable if it is possible to find a control sequence such that an arbitrary state can be reached from the origin in a finite time.

Definition 2. The linear discrete-time fractional-order system modeled by (37) is controllable if it is possible to find a control sequence such that the origin can be reached from any initial state in a finite time.

Definition 3. For the linear discrete-time fractional-order system modeled by (37) we define the following

1. The controllability matrix

$$
\mathcal{C}_{k}=\left[\begin{array}{lllll}
\mathrm{G}_{0} B & \mathrm{G}_{1} B & \mathrm{G}_{2} B & \cdots & \mathrm{G}_{k-1} B
\end{array}\right]
$$

2. The reachability Gramian

$$
\mathrm{W}_{r}(0, k)=\sum_{j=0}^{k-1} \mathrm{G}_{j} B B^{T} \mathrm{G}_{j}^{T}, \quad k \geq 1
$$

It is easy to show that $\mathrm{W}_{r}(0, k)=\mathcal{C}_{k} \mathcal{C}_{k}^{T}$.

3. The controllability Gramian, provided that $\mathrm{A}_{0}$ is non-singular

$$
\mathrm{W}_{c}(0, k)=\mathrm{G}_{k}^{-1} \mathrm{~W}_{r}(0, k) \mathrm{G}_{k}^{-T}, \quad k \geq 1
$$

Note that $G_{1}=A_{0}$ and the existence of $W_{r}(0,1)$ imposes $A_{0}$ to be non-singular. However, this is not that restrictive condition because a discrete model is often obtained by sampling a continuous one. Thus, in the remainder of this paper we assume that $A_{0}$ is non-singular.

Theorem 2. The linear discrete-time fractional-order system modeled by (37) is reachable if and only if there exists a finite time $K$ such that $\operatorname{rank}\left(\mathcal{C}_{K}\right)=n$ or, equivalently, $\operatorname{rank}\left(\mathrm{W}_{r}(0, K)\right)=n$. Furthermore, the input sequence

$$
\mathcal{U}_{K}=\left[\begin{array}{llll}
u^{T}(K-1) & u^{T}(K-2) & \ldots & u^{T}(0)
\end{array}\right]^{T}
$$

that transfers $x_{0}=0$ at $k=0$ to $x_{f} \neq 0$ at $k=K$ is given by

$$
\mathcal{U}_{K}=\mathcal{C}_{K}^{T} \mathrm{~W}_{r}^{-1}(0, K) x_{f}
$$


Remark 4. In the case of an integer order, it is well known that the rank of $\mathcal{C}_{k}$ cannot increase for any $k \geq n$. This results from the Cayley-Hamilton theorem. On the contrary, in the case of the linear discrete-time non-commensurate fractional-order system (37), the rank of $\mathcal{C}_{k}$ can increase for values of $k \geq n$. In other words, it is possible to reach the final state $x_{f}$ in a number of steps greater than $n$. This is due to the nature of the elements $\mathrm{G}_{k}$ which build up the controllability matrix $\mathcal{C}_{k}$ and which exhibit the particularity of being time-varying, in the sense that they are composed of a number of terms $\mathrm{A}_{j}$ that grows with $k$, as already mentioned in Remark 2. The full rank of $\left(\mathcal{C}_{k}\right)$ can be reached in some Step $k=K$ equal to, or greater than $n$.

Theorem 3. The linear discrete-time fractional-order system modeled by (37) is controllable if and only if there exists a finite time $K$ such that $\operatorname{rank}\left(\mathrm{W}_{c}(0, K)\right)=n$. Furthermore, an input sequence $\mathcal{U}_{K}=\left[\begin{array}{llll}u^{T}(K-1) & u^{T}(K-2) & \ldots & u^{T}(0)\end{array}\right]^{T}$ that transfers $x_{0} \neq 0$ at $k=0$ to $x_{f}=0$ at $k=K$ is given by

$$
\mathcal{U}_{K}=-\mathcal{C}_{K}^{T} \mathrm{G}_{K}^{-T} \mathrm{~W}_{c}^{-1}(0, K) x_{0}
$$

\subsection{Observability}

In this section we aim at extending the concept of observability to the system of Equations (37) and (38), in the case of a non-commensurate fractional-order. We are interested in completely state observable systems.

Definition 4. The linear discrete-time fractional-order system modeled by Equations (37) and (38) is observable at time $k=0$ if and only if there exits some $K>0$ such that the state $x_{0}$ at time $k=0$ can be uniquely determined from the knowledge of $u_{k}, y_{k}, k \in[0, K]$.

Definition 5. For the linear discrete-time fractional-order system modeled by Equations (37) and (38) we define the following

1. The observability matrix

$$
\mathcal{O}_{k}=\left[\begin{array}{c}
C G_{0} \\
C G_{1} \\
C G_{2} \\
\vdots \\
C G_{k-1} \cdot
\end{array}\right]
$$

2. The observability Gramian

$$
\mathrm{W}_{o}(0, k)=\sum_{j=0}^{k-1} \mathrm{G}_{j}^{T} C^{T} C \mathrm{G}_{j} .
$$

It is easy to show that $\mathrm{W}_{o}(0, k)=\mathcal{O}_{k}^{T} \mathcal{O}_{k}$.

Theorem 4. The linear discrete-time fractional-order system modeled by Equations (37) and (38) is observable if and only if there exists a finite time $K$ such that $\operatorname{rank}\left(\mathcal{O}_{K}\right)=n$ or, equivalently, $\operatorname{rank}\left(\mathrm{W}_{o}(0, K)\right)=n$. Furthermore, the initial state $x_{0}$ at $k=0$ is given by

$$
x_{0}=\mathrm{W}_{o}^{-1}(0, K) \mathcal{O}_{K}^{T}\left[\tilde{\mathcal{Y}}_{K}-\mathcal{M}_{K} \tilde{\mathcal{U}}_{K}\right]
$$


with

$$
\begin{aligned}
& \tilde{\mathcal{U}}_{K}=\left[\begin{array}{llll}
u^{T}(0) & u^{T}(1) & \ldots & u^{T}(K-1)
\end{array}\right]^{T}, \\
& \tilde{\mathcal{Y}}_{K}=\left[\begin{array}{llll}
y^{T}(0) & y^{T}(1) & \ldots & y^{T}(K-1)
\end{array}\right]^{T},
\end{aligned}
$$

and

$$
\mathcal{M}_{K}=\left[\begin{array}{cccccc}
0 & 0 & 0 & \ldots & 0 & 0 \\
C G_{0} B & 0 & 0 & \ldots & 0 & 0 \\
C G_{1} B & C G_{0} B & 0 & \ldots & 0 & 0 \\
C G_{2} B & C G_{1} B & C G_{0} B & \ldots & 0 & 0 \\
\vdots & \vdots & \vdots & \ldots & \vdots & \vdots \\
C G_{K-2} B & C G_{K-3} B & C G_{K-4} B & \ldots & C G_{0} B & 0
\end{array}\right] .
$$

Remark 5. From the Cayley-Hamilton theorem, it is well known that for integer-order systems the rank of the observability matrix $\mathcal{O}_{k}$ cannot increase in Step $k \geq n$. Here too, it is remarkable that this is not true in the case of the discrete-time non-commensurate fractional-order system of (37) and (38). Indeed, $\operatorname{rank}\left(\mathcal{O}_{k}\right)$ can increase for values $k \geq n$. We can state that the observability of this type of systems can possibly be obtained in a number of steps greater than $n$. This is due to the same reasons as those exposed above in Remark 3 for controllability. In [53], the observability condition for the discrete-time fractional-order system as modeled by (37), with non-commensurate order, is that the rank of $\mathcal{O}_{k}$ should be equal to $n$ at most in Step $k=n$. Our result shows that the full rank of $\left(\mathcal{O}_{k}\right)$ can be reached in some Step $k=K$ greater than $n$. This can be considered as an extension of the previous result in [53].

Commensurate fractional-order case In this section we address the particular case of commensurate FOS. The terms $A_{j}$ are as expressed by $A_{j}=-c_{j+1} \mathbb{I}_{n}$, for all $j>0$. It is clear then that matrices $G_{k}$ defined by (40) are polynomials in $A_{0}$, i.e.,

$$
G_{k}=A_{0}^{k}+\beta_{1_{k}} A_{0}^{k-1}+\beta_{2_{k}} A_{0}^{k-2}+\ldots+\beta_{k_{k}} \mathbb{I}_{n}
$$

where the real coefficients $\beta_{j_{k}}$ are calculated from the coefficients $c_{j}$. In particular, we have

$$
G_{n}=A_{0}^{n}+\beta_{1_{n}} A_{0}^{n-1}+\beta_{2_{n}} A_{0}^{n-2}+\ldots+\beta_{n_{n}} \mathbb{I}_{n}
$$

From the Cayley-Hamilton theorem, $A_{0}^{n}$ is a linear combination of $A_{0}^{n-1}, A_{0}^{n-2}, \ldots, \mathbb{I}_{n}$. We deduce that $G_{k+n}$, for all $k \geq 0$ are linearly dependent on $G_{n-1}, G_{n-2}, \ldots, \mathbb{I}_{n}$. This implies the following results

Corollary 1. The linear discrete-time fractional-order system modeled by Equations (37) and (38) in the commensurate case is reachable if and only if $\operatorname{rank}\left(\mathcal{C}_{n}\right)=n$ or, equivalently, $\operatorname{rank}\left(\mathrm{W}_{r}(0, n)\right)=n$. On the other hand, this system is controllable if and only if $\operatorname{rank}\left(\mathrm{W}_{\mathcal{c}}(0, n)\right)=n$.

Corollary 2. The linear discrete-time fractional-order system modeled by (37) and (38) in the commensurate case is observable if and only if $\operatorname{rank}\left(\mathcal{O}_{n}\right)=n$ or, equivalently, $\operatorname{rank}\left(\mathrm{W}_{o}(0, n)\right)=n$. 
Remark 6. We therefore observe that the controllability and observability criteria for the commensurate fractional-order case are similar to those of the integer-order case, in the sense that if a state cannot be reached in $n$ steps, then it is not reachable at all and that if an initial state cannot be deduced from $n$ steps of input-output data, then it is not observable at all. The result put forward in [53] which states that a necessary and sufficient condition for the discrete-time fractional-order system as modeled in (37) and (38) to be observable is that the rank of $\mathcal{O}_{k}$ should be equal to $n$ at most in Step $k=n$ is true only in the case of commensurate FOS.

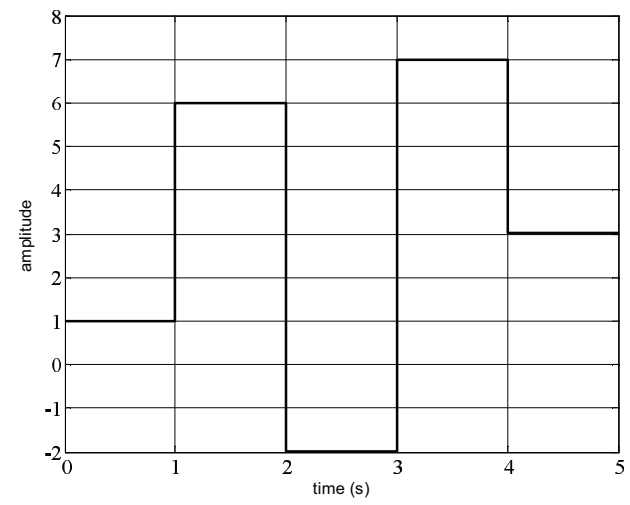

Figure 1. Computed values of the output sequence

\subsection{Stability analysis}

In this section, we investigate the property of stability of linear discrete-time FOS. We know that the study of the stability of FOS is limited to the result of the "'argument principle"', applicable to the sole category of commensurate-order systems. As far as non-commensurate orders are considered in the new models introduced, other adequate tools are required. In this context, new results concerning asymptotic stability are developed below. Besides, we introduce the concept of practical stability and we establish some mathematical conditions to check this property.

Asymptotic stability Let us consider the state-space model given by (37) and (38)

$$
\begin{gathered}
x(k+1)=\sum_{j=0}^{k} A_{j} x(k-j)+B u(k), \quad x(0)=x_{0}, \\
y(k)=C x(k),
\end{gathered}
$$

Its solution, given by $(40)$ is

$$
x(k)=G_{k} x_{0}+\sum_{j=0}^{k-1} G_{k-1-j} B u(j) .
$$


In order to study the asymptotic stability property of this system, we consider its unforced version, i.e.,

$$
x(k+1)=\sum_{j=0}^{k} A_{j} x(k-j), \quad x(0)=x_{0} .
$$

Definition 6. System (54) is asymptotically stable if for each $k \geq 1$ and any initial condition $x_{0}$, the following equality is verified

$$
\lim _{k \rightarrow \infty}\|x(k)\|=0
$$

We use the 2-norm of the state vector $x(k)$, i.e.,

$$
\|x(k)\|=\sqrt{\sum_{i=1}^{n} x_{i}^{2}(k)},
$$

where $x_{i}(k)$ are the components of $x(k)$.

The solution to (54) for given initial conditions $x_{0}$ is

$$
x(k)=G_{k} x_{0}, \quad k \geq 1, G_{0}=\mathbb{I}_{n} .
$$

It follows that System (54) is asymptotically stable if and only if

$$
\left\|G_{k}\right\| \leq 1, \quad k \geq 1
$$

The 2-norm of the transition matrix is

$$
\left\|G_{k}\right\|=\lambda_{\text {max }}^{\frac{1}{2}}\left(G_{k} G_{k}^{T}\right)=\sigma_{\max }\left(G_{k}\right)
$$

where $\lambda_{\max }$ and $\sigma_{\max }$ refer to the maximal eigenvalue and the maximal singular value of $G_{k}$ respectively.

Let us define the backward shift operator $\mathcal{S}$ as follows ([55], [56]). We consider an infinite sequence of samples of vector $x$, denoted $\mathbf{x}$, starting from $k=0$ up to infinity, and with null values for $k<0$, assuming the system to be causal. Thus, we have

$$
\mathbf{x}=\{\ldots, 0,0, x(0), x(1), x(2), \ldots, x(N), \ldots\}
$$

$\mathcal{S}$ acts on $\mathrm{x}$ as follows

$$
\begin{aligned}
\mathcal{S} \mathbf{x} & =\mathcal{S}\{\ldots, 0,0, x(0), x(1), x(2), \ldots, x(k), x(k+1), \ldots\} \\
& =\{\ldots, 0,0,0,0, x(0), x(1), x(2), \ldots, x(k-1), \ldots\} .
\end{aligned}
$$


We then define the column sequence

$$
\widetilde{\mathbf{x}}=\left[\begin{array}{c}
\vdots \\
0 \\
x(0) \\
x(1) \\
\vdots \\
x(k) \\
\vdots
\end{array}\right]
$$

Similarly, we can use this representation to rewrite (37) and its output equation

$$
y(k)=C x(k)
$$

in the equivalent form

$$
\begin{gathered}
\widetilde{\mathbf{x}}=\widetilde{\mathbf{S}} \widetilde{\mathbf{A}} \widetilde{\mathbf{x}}+\widetilde{\mathbf{S}} \widetilde{\mathbf{B}} \widetilde{\mathbf{u}}, \\
\widetilde{\mathbf{y}}=\widetilde{\mathbf{C}} \widetilde{\mathbf{x}} .
\end{gathered}
$$

In this representation, the expressions of the different components are

$$
\widetilde{\mathbf{u}}=\left[\begin{array}{c}
\vdots \\
u(0) \\
u(1) \\
\vdots \\
u(k) \\
\vdots
\end{array}\right], \quad \widetilde{\mathbf{y}}=\left[\begin{array}{c}
\vdots \\
0 \\
y(0) \\
y(1) \\
\vdots \\
y(k) \\
\vdots
\end{array}\right], \quad \widetilde{\mathbf{s}}=\left[\begin{array}{cccc}
0 & 0 & 0 & \ldots \\
\mathbb{I}_{n} & 0 & \ldots \ldots \ldots \\
0 & \mathbb{I}_{n} & \ldots \ldots \\
0 & 0 & \mathbb{I}_{n} & \ldots \ldots \\
\vdots & \vdots & \vdots & \vdots
\end{array}\right]
$$

and

$$
\widetilde{\mathbf{A}}=\left[\begin{array}{cccc}
A_{0} & 0 & 0 & 0 \ldots \\
A_{1} & A_{0} & 0 & 0 \ldots \\
A_{2} & A_{1} & A_{0} & 0 \ldots \\
A_{3} & A_{2} & A_{1} & A_{0} \ldots \\
\vdots & \vdots & \vdots & \vdots
\end{array}\right], \quad \widetilde{\mathbf{B}}=\left[\begin{array}{ccccc}
B & 0 & 0 & 0 & \ldots \\
0 & B & 0 & 0 & \ldots \\
0 & 0 & B & 0 & \ldots \\
0 & 0 & 0 & B & \ldots \\
\vdots & \vdots & \vdots & \vdots & \vdots
\end{array}\right]
$$

and 


$$
\widetilde{\mathbf{C}}=\left[\begin{array}{ccccc}
C & 0 & 0 & 0 & \ldots \\
0 & C & 0 & 0 & \ldots \\
0 & 0 & C & 0 & \ldots \\
0 & 0 & 0 & C & \ldots \\
\vdots & \vdots & \vdots & \vdots & \vdots
\end{array}\right]
$$

Theorem 5. Putting $\mathcal{A}_{s}=\widetilde{\mathbf{S}} \widetilde{\mathbf{A}}$, the system

$$
\widetilde{\mathbf{x}}=\widetilde{\mathbf{S}} \widetilde{\mathbf{A}} \widetilde{\mathbf{x}}=\mathcal{A}_{S} \widetilde{\mathbf{x}}
$$

is asymptotically stable if and only if $\rho\left(\mathcal{A}_{s}\right) \leq 1$, where $\rho$ is the spectral radius of operator $\mathcal{A}_{s}$, defined as

$$
\rho\left(\mathcal{A}_{s}\right)=\lim _{i \rightarrow \infty}\left\|\mathcal{A}_{S}^{i}\right\|_{*}{ }^{\frac{1}{i}}
$$

in which the norm definition is

$$
\left\|\mathcal{A}_{s}^{i}\right\|_{*}=\sup _{[I, J]}\left\|\mathcal{A}_{s[I, J]}^{i}\right\|
$$

with $\mathcal{A}_{s[I, J]}^{i}$ denoting the $[I, J]^{\text {th }}$ block matrix of $\mathcal{A}_{s}^{i}$.

The proof of this theorem is in [36]. In practice, a finite-time observation of the system is desirable. For this purpose, we consider the concept of practical stability ([54], [57]) defined as follows

Definition 7. System (54) is practically stable in a finite-time horizon $L_{h}>0$ if for each $1 \leq k \leq L_{h}$ and any initial condition $x_{0}$ the following inequality is verified

$$
\|x(k)\| \leq M\left\|x_{0}\right\|
$$

where $M$ is a strictly positive finite given number.

From solution (56), System (54) is practically stable if and only if $\left\|G_{k}\right\| \leq M$ for $1 \leq k \leq L$. Besides, we can write

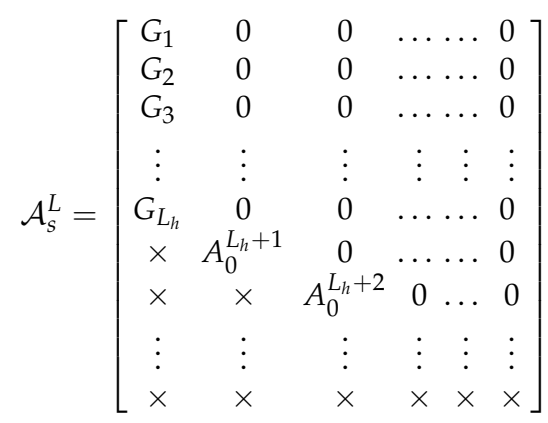


Define the block matrix

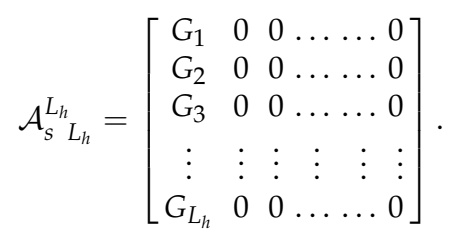

From the above, we can state that System (54) is practically stable if and only if $\left\|\mathcal{A}_{s}^{L_{h} L_{h}}\right\|_{*} \leq$ $M$. These results are illustrated by a numerical example in [36].

Remark 7. The state-space representations elaborated in the previous sections for the continuous time and the discrete time were considered for a SISO process. They are easily extended to Multiple Input, Multiple Output (MIMO) systems, with the use of a vector of inputs u instead of a scalar input, and a vector of outputs $y$ instead of a scalar output. MIMO systems are treated with a same procedure. This enables the study of their structural properties and permits to deal with control issues of such systems either in time domain or frequency domain, such as MIMO $\mathrm{H}_{\infty}$ problem for example in[58].

\section{Practical example}

\subsection{Fractional-order modeling of a thermal system}

We consider a thermal system consisting in the regulation of the temperature profile of an aluminium rod with constant thermal conductivity $\kappa$ and diffusivity $\xi$, as described in [30, 59]. A possible version is shown in Figure 2. Temperature $T(\chi, t)$ is evaluated at abscissa $\chi$

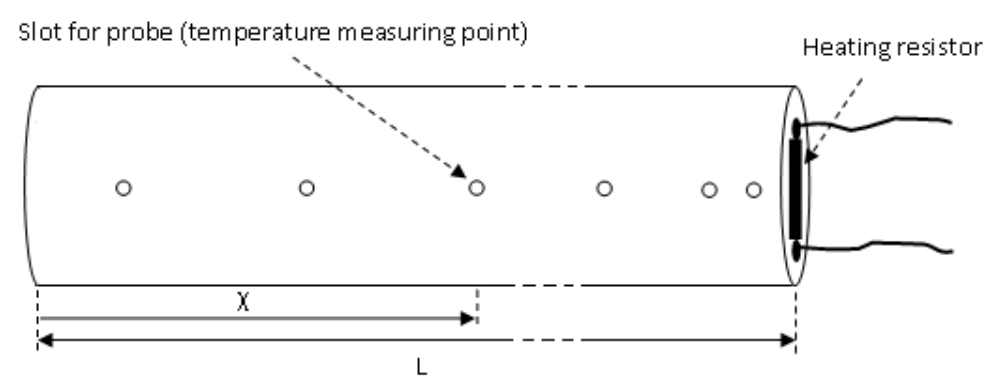

Figure 2. The metallic rod thermal system.

along the rod axis. The rod length is $L$, and the origin is set at the non heated end. Measuring points of $T(\chi, t)$, by means of probes, are provided at different distances. To build a model of this system, we use an approximation by considering that the temperature diffusion for this type of geometry is governed by the following partial differential equation (PDE)

$$
\frac{\partial T(\chi, t)}{\partial t}=\xi \frac{\partial^{2} T(\chi, t)}{\partial \chi^{2}}, \quad \chi \in(0, L), \quad t \geq 0
$$


The Dirichlet boundary condition is stated as follows [59]: we consider the control action that maintains a temperature $u(t)$ fixed at the end point $\chi=L$ and that a temperature measurement can be taken inside the rod, at a fixed abscissa $\chi_{0}$ (see Figure 2). Therefore, the boundary conditions are

$$
T(0, t)=0 . \quad T(L, t)=u(t) .
$$

As for the temperature measured at a fixed point $\chi_{0}$, this represents the observation, that is

$$
y(t)=T\left(\chi_{0}, t\right)
$$

\subsection{Fractional-order state-space model of the aluminium rod}

Let us denote the Laplace transforms of $T(\chi, t)$ and $u(t)$ respectively by $\bar{T}(\chi, s)$ and $\bar{u}(s)$. The assumption of null initial conditions is made here, i.e., the rod is left to cool down before experiment. This conducts us to an easier computing process. Indeed non null initial conditions in the fractional case, as exposed for example in [31], conduct to computations that are more complicated to handle. Equation (63) and (64) become

$$
\begin{aligned}
& \frac{d^{2} \bar{T}(\chi, s)}{d \chi^{2}}=\frac{s}{\xi} \bar{T}(\chi, s)=0, \quad \text { with } \\
& \bar{T}(0, s))=0, \quad \bar{T}(L, s)=\bar{u}(s) .
\end{aligned}
$$

The corresponding solution leads to the transfer function

$$
H\left(\chi_{0}, s\right)=\frac{\bar{y}(s)}{\bar{u}(s)}=\frac{\bar{T}\left(\chi_{0}, s\right)}{\bar{T}(L, s)}=\frac{\sinh \left(\chi_{0} \sqrt{\frac{s}{\bar{\zeta}}}\right)}{\sinh \left(L \sqrt{\frac{s}{\bar{\zeta}}}\right)}
$$

This expression establishes that the described device exhibits the feature of fractional-order model. It is the case for various other types of diffusion, say chemical diffusion processes, neutron flux, and also for dielectric relaxation, electrode polarization, transmission lines, etc.. The four $\exp ($.$) terms composing the last part of (67) are expressed by using a Padé$ approximation of order 2 . Let us recall here that, for instance, for $\exp ($.$) with given argument$ $z$, a Padé approximation of order $P$ yields

$$
\exp (z) \approx \frac{\sum_{k=0}^{P} \frac{(2 P-k) !}{k !(P-k) !}(z)^{k}}{\sum_{k=0}^{P} \frac{(2 P-k) !}{k !(P-k) !}(-z)^{k}}
$$

The determination of $H\left(\chi_{0}, s\right)$ is made for an aluminium rod, with a set of numerical values most of them used in [30]. These are

- $\operatorname{rod}$ length $L=0.4 \mathrm{~m}$. 
- abscissa of the measuring point, set here at $\chi_{0}=\frac{2 * L}{3} \mathrm{~m}$.

- $\quad$ rod thermal conductivity $\kappa=237 \mathrm{~W} / \mathrm{m} . \mathrm{K}$, and diffusivity $\xi=9975.10^{-8} \mathrm{~m}^{2} / \mathrm{s}$.

With this set of practical values, we obtain the following transfer function

$$
H\left(\chi_{0}, s\right)=\frac{0.6667+22.2501 s^{\frac{1}{2}}+188.1246 s-1982.7 s^{\frac{3}{2}}+5293.9 s^{2}}{1+33.3751 s^{\frac{1}{2}}+430.7064 s+1982.7 s^{\frac{3}{2}}+3529.3 s^{2}}
$$

Next we apply to (68) the procedure exposed above from (8) to (17), so as to obtain the corresponding fractional-order state-space model. We find a state-space model with commensurate fractional orders, of the form (17). In our case, it can be reduced to four states, by eliminating redundant states through a variable change. In the resulting model, there exists a direct transmission matrix $D$, what can be foreseen when considering the monomials of highest degree in $s$ of numerator and denominator of (68).

The computed set of matrices $A, B, C, D$ of the resulting fractional-order state-space model for the rod, in continuous time, is therefore

$$
\begin{gathered}
A=\left[\begin{array}{cccc}
0 & 1 & 0 & 0 \\
0 & 0 & 1 & 0 \\
0 & 0 & 0 & 1 \\
-0.0003 & -0.0095 & -0.1220 & -0.5618
\end{array}\right], \quad B=\left[\begin{array}{c}
0 \\
0 \\
0.0002833
\end{array}\right], \\
C=[-0.8333-27.8126-457.9350-4956.8], \quad D=[1.5] .
\end{gathered}
$$

\subsection{Analysis of the discrete-time state-space model of the rod}

We convert the continuous-time state space model described by the set of matrices (69) into a discrete-time version. This is done by choosing the sampling period $h$, making $h=1 \mathrm{~s}$. The sampling period value taken in [30] for the same aluminium rod is somewhat smaller, that is $0.5 \mathrm{~s}$. We set the input variable $u(t)=T(L, t)=320^{\circ} \mathrm{K}$. In other words, the temperature at abscissa $\chi=L$ is maintained constant. The observed output $y(t)=T\left(\chi_{0}, t\right)$ is the temperature diffusing at a measuring point at $\chi_{0}$. The response obtained is shown in Figure 3. It indicates that the output temperature starts increasing from null initial condition set at $T(0, t)=0^{\circ} \mathrm{K}$, which is an assumption for deriving the model used. In the interval of the experiment over 3000 steps, it diffuses and tends to a value approaching the input fixed value. A detailed plot represented in Figure 4 shows at the beginning an increase of $y(t)$, followed by a brief fall under zero. This type of behaviour at first sight is similar to non-minimum phase system behaviour. However, its interpretation as a physical phenomenon appears to be not realistic, considering the underlying process in study.

Besides, as an example of study of the structural properties of the model proposed, we have led a reachability test, following the theoretical developments of previous Section 4 . Starting from a zero initial state $x(0)=\left[\begin{array}{llll}0 & 0 & 0 & 0\end{array}\right]^{\prime}$, we set a final state to be reached as $x_{f}=\left[\begin{array}{llll}-0.13 & -0.13 & -0.13 & -0.13\end{array}\right]^{\prime}$. The computation achieved yields the result that $x_{f}$ is exactly reached in four steps, i.e. in a number of steps equal to the rank of the controllability matrix, as depicted in Figure 5. Nevertheless, from the point of view of 
physical coherence, the result is not satisfactory, since the necessary input sequence yielded is $u(k)=\left[\begin{array}{llll}-278.2396 & -99.607 & -36.5687 & -18.3551\end{array}\right]$, that is, with (all) negative elements. Related to this feature, the values of the model output matrix $C$ is found to be made of all negative elements. A further insight should be therefore directed towards the modeling process, initiated with the representation of the diffusive phenomena by PDE equations. Next, a more detailed evaluation of the precision when achieving the Padé approximation would be expectable. It remains that one major improvement to expect is the effective introduction in the modeling of fractional-order systems of non null initial conditions [31]. Taking them into account in the computations is a way to make the representations closer to the reality of the phenomena. The case treated here, which aims to describe the diffusion of a given fixed temperature at one end of the rod, implies to deal with realistic operation conditions. For example, an interesting case to investigate would be to consider the rod set initially at ambient temperature, say currently around $300{ }^{\circ} \mathrm{K}$, as a non null initial condition, and to track the diffusion of a given input temperature with a more elaborated model.

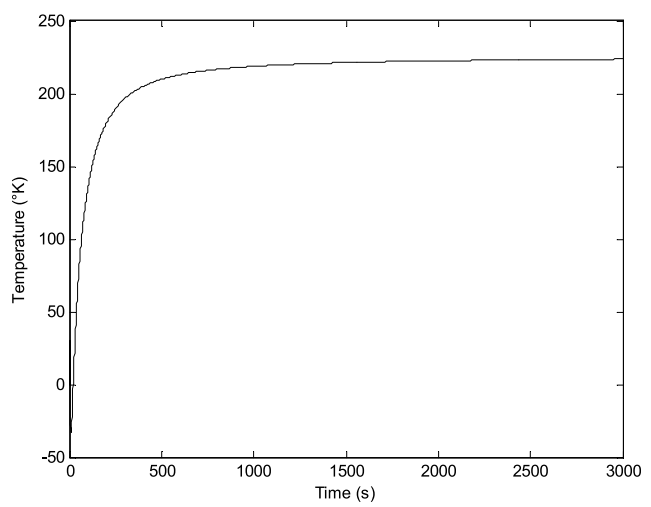

Figure 3. Output temperature evolution over 3000 points (Sampling period: 1s).

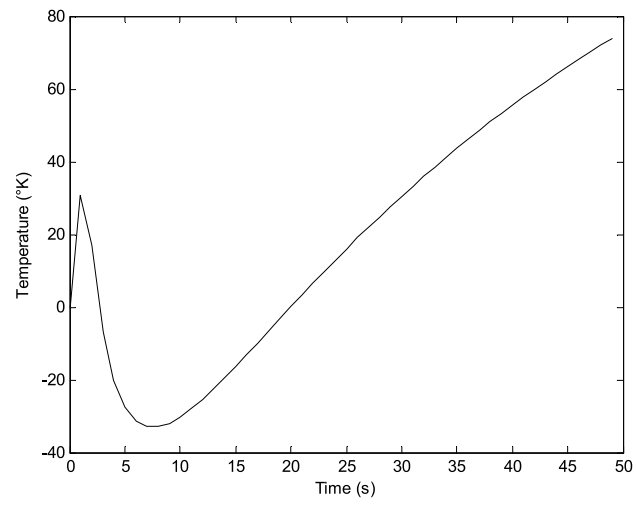

Figure 4. Output temperature evolution, starting from zero initial condition (Detail). 


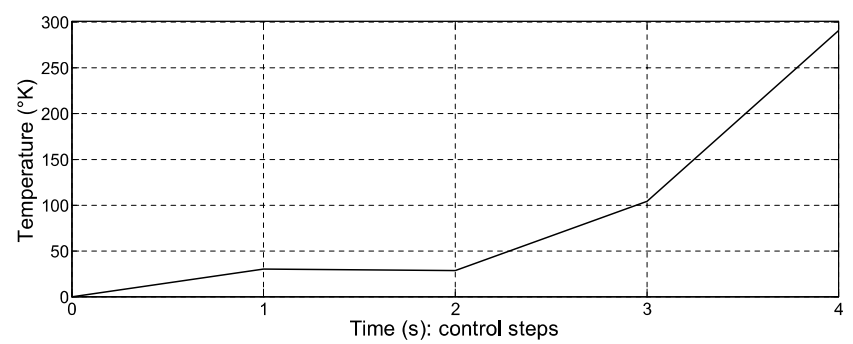

Figure 5. Output temperature evolution during control sequence.

\section{Stabilization issue}

In this section, we deal with the problem of stabilization. For this purpose, we consider the system given by (37) and (38), which we aim to stabilize, by handling state equation (37)

$$
x(k+1)=\sum_{j=0}^{k} A_{j} x(k-j)+B u(k), \quad x(0)=x_{0} .
$$

Let us consider the control $u$ as a state-feedback control, in a classical state-feedback control loop, with a null reference input $(r=0)$, as illustrated in Figure 6. We assume that

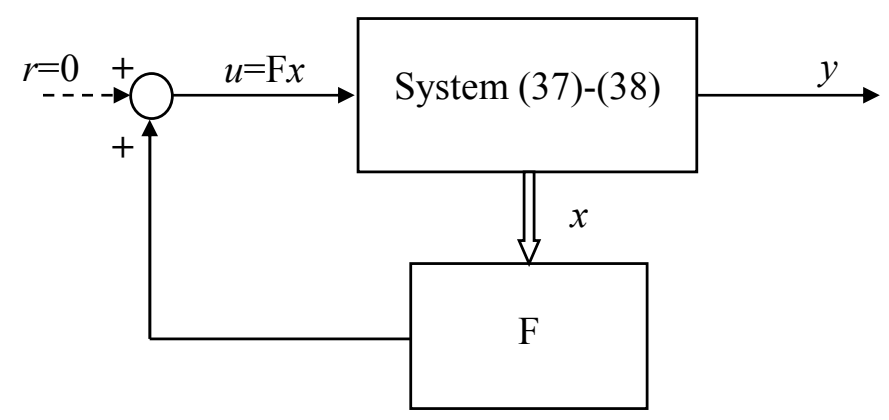

Figure 6. State-feedback control loop.

$$
u(k)=\sum_{j=0}^{k} F_{j} x(k-j)
$$

That is

$$
u(k)=F_{0} x(k)+F_{1} x(k-1)+F_{2} x(k-2)+\ldots+F_{k} x(0) .
$$


In the closed loop, we have

$$
x(k+1)=\sum_{j=0}^{k}\left(A_{j}+B F_{j}\right) x(k-j) .
$$

Then, putting $A_{F j}=A_{j}+B F_{j}$, we can write

$$
x(k+1)=\sum_{j=0}^{k} A_{F j} x(k-j) .
$$

Thus, successively, we build the recurrence beginning with

$$
\begin{gathered}
x(1)=A_{F 0} x(0)=\left(A_{0}+B F_{0}\right) x(0), \\
x(2)=A_{F 0} x(1)+A_{F 1} x(0)=\left(A_{0}+B F_{0}\right) x(1)+\left(A_{1}+B F_{1}\right) x(0), \\
x(3)=A_{F 0} x(2)+A_{F 1} x(1) A_{F 2} x(0)=\left(A_{0}+B F_{0}\right) x(2)+\left(A_{1}+B F_{1}\right) x(1)+\left(A_{2}+B F_{2}\right) x(0), \ldots
\end{gathered}
$$

This leads to the following relation between the two column sequences

$$
\left[\begin{array}{c}
x(1) \\
x(2) \\
x(3) \\
\vdots \\
x(k+1) \\
\vdots
\end{array}\right]=\left[\begin{array}{cccc}
\left(A_{0}+B F_{0}\right) & 0 & 0 & 0 \ldots \\
\left(A_{1}+B F_{1}\right) & \left(A_{0}+B F_{0}\right) & 0 & 0 \ldots \\
\left(A_{2}+B F_{2}\right) & \left(A_{1}+B F_{1}\right) & \left(A_{0}+B F_{0}\right) & 0 \ldots \\
\vdots & \vdots & \vdots & \vdots
\end{array}\right]\left[\begin{array}{c}
x(0) \\
x(1) \\
x(2) \\
\vdots \\
x(k) \\
\vdots
\end{array}\right]
$$

Similarly to the notations used in (37), i.e., considering an infinite column sequence $\widetilde{\mathbf{x}}$, and using the backward shift $\mathcal{S}$, Equation (58) is rewritten as follows

$$
\widetilde{\mathbf{x}}=\widetilde{\mathbf{S}} \widetilde{\mathbf{A}_{\mathbf{F}}} \widetilde{\mathbf{x}}
$$

in which

$$
\widetilde{\mathbf{A}_{\mathbf{F}}}=\left[\begin{array}{cccc}
\left(A_{0}+B F_{0}\right) & 0 & 0 & 0 \ldots \\
\left(A_{1}+B F_{1}\right) & \left(A_{0}+B F_{0}\right) & 0 & 0 \ldots \\
\left(A_{2}+B F_{2}\right) & \left(A_{1}+B F_{1}\right) & \left(A_{0}+B F_{0}\right) & 0 \ldots \\
\vdots & \vdots & \vdots & \vdots
\end{array}\right]
$$

Further, we decompose $\widetilde{\mathbf{A}_{\mathbf{F}}}$ as follows 


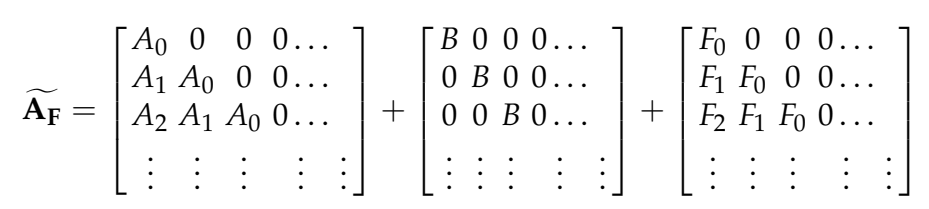

Equation (58) becomes

$$
\widetilde{\mathbf{x}}=\widetilde{\mathbf{S}} \widetilde{\mathbf{A}_{\mathbf{F}}} \widetilde{\mathbf{x}}=\widetilde{\mathbf{S}} \widetilde{\mathbf{A}} \widetilde{\mathbf{x}}+\widetilde{\mathbf{S}} \widetilde{\mathbf{B}} \widetilde{\mathbf{x}}
$$

where

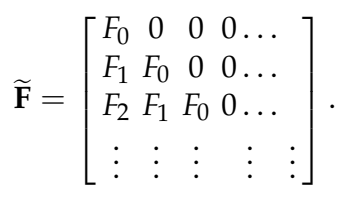

The reachability is reformulated here under the operator-theoretic formulation (Equations (58) and (59)) of the discrete-time fractional-order system.

Definition 8. The pair $(\widetilde{\mathbf{A}}, \widetilde{\mathbf{B}})$ is K-step reachable if the operator reachability Gramian defined by

$$
\widetilde{\mathbf{W}_{\mathbf{r}}^{\mathbf{p}}}=\widetilde{\mathbf{C}}_{p}^{T} \widetilde{\mathbf{C}}_{p}
$$

where

$$
\widetilde{\mathbf{C}}_{p}=\left[\begin{array}{lll}
\widetilde{\mathbf{B}} & \widetilde{\mathbf{A}} \widetilde{\mathbf{B}} \quad \ldots \widetilde{\mathbf{A}}^{p-1} \widetilde{\mathbf{A}}
\end{array}\right]
$$

is invertible.

Similarly to Equation (39), let us now define the transition matrix form $G_{k}$ such that

$$
G_{F k}=\left\{\begin{array}{cc}
\mathbb{I}_{n_{d}} & \text { for } k=0 \\
\sum_{j=0}^{k-1} A_{F j} G_{F(k-1-j)} & \text { for } k \geq 1
\end{array}\right.
$$

We now consider the problem of choosing the state feedback operator gain $\widetilde{\mathbf{F}}$ that stabilizes System (77), i.e., which ensures that

$$
\left\|G_{F k}\right\| \leq 1 \text { for } k \geq 1
$$

The following statement is derived from Theorem 5 established above, and Lemma 2 in [60]. Corollary 3. Suppose that $(\widetilde{\mathbf{A}}, \widetilde{\mathbf{B}})$ is K-reachable and let

$$
\widetilde{\mathbf{F}}=-\widetilde{\mathbf{B}}^{T} \widetilde{\mathbf{A}}^{T}\left(\widetilde{\mathbf{W}}_{r}^{p}\right)^{-1} \widetilde{\mathbf{A}}^{p+1},
$$

then the closed-loop system (77) is asymptotically stable, that is $\rho\left(\widetilde{\mathbf{A}}_{F s}\right)<1$, where $\widetilde{\mathbf{A}}_{F s}=\widetilde{\mathbf{S}} \widetilde{\mathbf{A}}_{F}$ 
Remark 8. The state feedback (70) uses the entire memory of the state variable. In practice, this could be undesirable and computationally unwieldy. It is preferable to design a controller with short-memory [45]. Let be $L_{h}$ the restricted length of the memory (or horizon). The practical state feedback is formulated as:

$$
u(k)=\sum_{j=0}^{L_{h}} F_{j} x(k-j), \quad \text { for } k \geq L_{h},
$$

and

$$
u(k)=\sum_{j=0}^{k} F_{j} x(k-j), \quad \text { for } k<L_{h}
$$

Stability of the short-memory state feedback controller could be investigated with the same development given in this section.

\section{Conclusion}

We have reviewed some tools for modeling and analysis of FOS in discrete time, introducing state-space representation for both commensurate and non commensurate fractional orders. These latter new approaches of modeling and analysis of such systems have revealed new properties, not shown in continuous time representations.

Our contribution concerns the analysis of the controllability and the observability of linear discrete-time FOS. We have introduced a new formalism and established testable sufficient conditions for guaranteeing the controllability and the observability. Some aspects of controllability and observability of such systems had not been treated before. Let us recall the remarkable point that, in the case of the linear discrete-time non-commensurate FOS, the rank of the controllability matrix can increase for values greater than the dimension of the system. In other words, it is possible to reach the final state in a number of steps greater than this dimension number. They are expected to give birth to further investigations and applications. With the use of a new formalism, an approach to analysis of asymptotic stability and practical stability of discrete-time FOS has been proposed.

The modeling of a practical system has been treated, which points out theoretical assumptions to match with real conditions.

Finally, the preliminary results presented in this chapter enabled us to make first steps into investigation on stabilization and practical stabilization of linear discrete-time FOS.

\section{Author details}

Saïd Guermah ${ }^{1}$,

Saïd Djennoune ${ }^{1}$ and Maâmar Bettayeb ${ }^{2}$

1 Laboratoire de Conception et Conduite des Systèmes de Production (L2CSP), Faculty of Electrical Engineering and Computer Science, Mouloud Mammeri University of Tizi-Ouzou, Tizi-Ouzou, Algeria

2 Department of Electrical/Electronics \& Computer Engineering, University of Sharjah, United Arab Emirates 


\section{References}

[1] Oldham KB, Spanier J. The fractional calculus. Academic Press. New-York; 1974.

[2] Miller KS, Ross B. An introduction to the fractional calculus and fractional differential equations, Wiley, New-York, 1993.

[3] Samko SG, Kilbas AA, Marichev OI. Fractional integrals and derivatives: theory and applications. Gordon and Breach Science Publisher, Amsterdam, 1993.

[4] Kilbas AA, Srivasta HM, Trujillo JJ. Theory and applications of fractional differential equations, Noth-Holland Mathematics Studies 204, Elsevier, Amsterdam, 2006.

[5] Manabe S. The non-Integer Integral and its Application to Control Systems, ETJ of Japan, 1961; 6 (3/4): 83-87.

[6] Mandelbrot B. The fractal geometry of nature, Freeman, San Fransisco; 1982.

[7] Charef A, Sun HH, Tsao YY, Onaral B. Fractal system as represented by singularity function, IEEE Transactions on Automatic Control, 1992; 37(9): 1465-1470.

[8] Oustaloup A. La dérivation non entière, théorie, synthèse et applications, Hermès Edition, Paris, 1995.

[9] Carpinteri A, Mainardi F. Fractals and Fractional Calculus in Continuum Mechanics, Springer Verlag, Vienna-New York, 1997.

[10] Montseny G. Diffusive representation of pseudo-differential time operator, Proceeding Fractional Differential Equation Systems: Model, Methods and Application, Paris, 1998.

[11] Battaglia JL, Le Lay L, Batsale JC, Oustaloup A, Cois O. Heat flux estimation through inverted non integer identification models, Int J of Thermal Science. 2000; 39 (3): 374-389.

[12] Caputo M. Distributed order differential equations modeling dielectric induction and diffusion, Fractional Calculus Appl. Anal. 2001; 4: 421-442.

[13] Heymans N. Implementation of fractional calculus using hierarchical models: application to the terminal transition of a complex polymer, in the Proc. of DETC 2003/VIB 48396 ASME, Chicago, USA, 2003.

[14] Zhang Yanzhu, Xue Dingyu. Wireless Communications, Networking and Mobile Computing, WiCom 2007.

[15] Ichise M, Nagayanagi Y, Kojima T. An analog simulation of non-integer order transfer functions for analysis of electrode processes, J of Electroanalytical Chemistry; 33: 253-265.

[16] Axtell M, Bise EM. Fractional calculus applications in control systems, in Proceedings of the IEE Nat. Aerospace and Electronics Conf.; 1990 New-York; 536-566. 
[17] Bagley RL, Calico RA. Fractional order state equations for the control of viscoelastically damped structures, J of Guidance, Control and Dynamics. 1991; 14: 304-311.

[18] Nakagava N, Sorimachi K. Basic characteristics of fractance device, IEICE Trans. Fund. 1992; E75-A (12):1814-1818.

[19] Matignon D, d'Andréa Novel B, Depalle P, Oustaloup A. Viscothermal losses in wind instrument: a non-integer model, Systems and Networks: mathematical theory and application. Academic Verlag Edition. 1994; 2.

[20] Bidan G. Commande diffusive d'une machine électrique: une introduction, Proceeding Fractional Differential Equation Systems: Model, Methods and Application, Paris; 1998.

[21] Oustaloup A, Sabatier J, Moreau X. From fractional robustness to the CRONE approach, Proceeding Fractional Differential Equation Systems: Model, Methods and Application; 1998; Paris.

[22] Ortigueira MD. Introduction to fractional linear systems, IEE proc. Image signal process; 2000 February; 147 (1).

[23] Cois O, Oustaloup A, Battaglia E, Battaglia JL. Non integer model from modal decomposition for time domain identification; 41 st IEEE CDC'2002 Tutorial Workshop 2; Las Vegas, USA

[24] Vinagre BM , Monje CA, Calderon AJ. Fractional order systems and fractional order actions. Tutorial Workshop 2: Fractional Calculus Applications in Automatic Control and Robotics; 2002; Las Vegas, USA.

[25] Hanyga A. Internal variable models of viscoelasticity with fractional relaxatioon laws. Proc. of DETC 2003/VIB, 48395, ASME, Chicago, USA.

[26] Moreau X, Altet O, Oustaloup A. The CRONE suspension: modeling and stability analysis. DETC, ASME; 2003 Sep 2-6; Chicago, Illinois, USA.

[27] Djouambi A, Charef A, Bouktir T. Fractal Robustness and Parameter Tuning $P I^{\lambda} D^{\mu}$ Controllers. Proc. of the 5th WSEAS Int. Conf. on Signal, Speech and Image Processing; 2005 Aug 17-19:155-162; Corfu, Greece.

[28] Heymans N, Podlubny I. Physical interpretation of initial conditions for fractional differential equations with Riemann-Liouville fractional derivatives, Rheol. Acta. 2006 (45):765-772.

[29] Battaglia JL, Cois O, Puigsegur L, Oustaloup A. Solving an inverse heat conduction problem using a non-integer identified model, Int J of Heat and Mass Transfer 2001; 44(14).

[30] Malti R, Sabatier J, Akçay H. Thermal modeling and identification of an aluminium rod using fractional calculus., 15th IFAC Symposium on System Identification (SYSID); 2009; Saint-Malo, France. 
[31] Hartley TT, Lorenzo CF. Dynamics and Control of Initialized Fractional-Order Systems. Nonlinear Dynamics. 2002; 29: 201-233.

[32] Monje CA, Chen YQ, Vinagre BM, Xue D, Feliu V. Fractional-order Systems and Controls: Fundamentals and Applications, ISSN 1430-9491; ISBN 978-1-84996-334-3, e-ISBN 978-1-84996-335-0, DOI 10.1007/978-1-84996-335-0, Springer London Dordrecht Heidelberg New York, 2010.

[33] Dzieliński A, Sierociuk D. Adaptive Feedback Control of Fractional Order Discrete State-Space Systems. Proc of the 2005 International Conference on Computational Intelligence for Modelling, Control and Automation, and International Conference on Intelligent Agents, Web Technologies and Internet Commerce (CIMCA-IAWTIC'05);524-529; 2005; Vienna, Austria.

[34] Guermah S, Djennoune S, Bettayeb M. Controllability and Observability of Linear Discrete-Time Fractional-Order Systems, Int J of Applied Mathematics and Computer Science (AMCS). 2008; 18(2):213-222.

[35] Guermah S, Djennoune S, Bettayeb M. State space analysis of linear fractional order systems. J Européen des Systèmes Automatisés. 2008; 42(6-7-8): 825-838.

[36] Guermah S, Djennoune S, Bettayeb M. A new approach for stability analysis of linear discrete-time fractional-order systems, in New Trends in Nanotechnology and Fractional Calculus Applications, Springer Book, Dimitru Baleanu, Zya Burhanettin, Güvenç JA, Tenreiro Machado Editors; 151-162; ISBN 978-90-481-3292-8, Springer, 2010. DOI 10.1007/978-90-481-3293-5/11

[37] Raynaud HF, Zergainoh A. State-space representation for fractional-order controllers. Automatica. 2000; 36: 1017-1021

[38] Hotzel R, Fliess M. On linear system with a fractional derivation: introductory theory and examples. Mathematics and Computers in Simulation. 1998; 45: 385-395

[39] Dorčák L, Petras I, Kostial I. Modeling and analysis of fractional-order regulated systems in the state-space. Proc. of ICCC'2000: 185-188; 2000; High Tatras, Slovak Republic.

[40] Sabatier J, Cois O, Oustaloup A. Commande de systèmes non entiers par placement de pôles. Deuxième Conférence Internationale Francophone d'Automatique, CIFA. 2002, Nantes, France.

[41] Mittag-Leffler G. Sur la représentation analytique d'une branche uniforme d'une fonction monogène, Acta Mathematica. 1904; 29: 10-181

[42] Matignon D. Stability results on fractional differential equations with application to control processing. In Computational Engineering in Systems Applications. 1996; 963-968 
[43] Matignon D, d'Andréa-Novel B. Some results on controllability and observability of finite-dimensional fractional differential systems. In IMACS, IEEE-SMC Proceedings Conference: 952-956; 1996; Lille, France.

[44] Bettayeb M, Djennoune S. A note on the controllability and the observability of fractional dynamical systems, Proceedings of the 2nd IFAC Workshop on Fractional Differentiation and its Applications (FDA'06):506-511; 2006 Jul 19-21; Porto, Portugal.

[45] Podlubny I. Fractional Differential Equations, Academic Press, New York, 1999.

[46] Dzieliński A, Sierociuk D. Reachability, controllability and observability of the fractional order discrete state-space system, IEEE/IFAC International Conference on the Methods and Models in Automation and Robotics, MMAR'2007; 2007 Aug 27-30; Szczecin, Poland.

[47] Lakshmikantham DTV. Theory of Difference Equations: Numerical Methods and Applications, Academic Press, New-York, 1988.

[48] Aström KJ, Wittenmark B. Computer-controlled systems, Theory and design, 2nd edition, Prentice Hall, Englewoods Cliffs, New Jersey, 1990.

[49] Billings SA, Aguirre LA (University of Sheffield, Department of Automatic Control and Systems Engineering, Sheffield, UK). Effects of the Sampling Time on the Dynamics and Identification of Nonlinear Models. Research report 513; Sheffield, 1994.

[50] Peng Y, Guangming X, Long W. Controllability of Linear Discrete-Time Systems With Time-Delay in State, In dean.pku.edu.cn/bksky/1999tzlwj/4.pdf., 2003.

[51] Boukas EK. Discrete-Time Systems with Time-varying Time Delay: Stability and Stabilizability. Hindawi Publishing Corporation, Mathematical Problems in Engineering. 2006; 2006:1-10

[52] Debeljković DL, Aleksendrić M, Yi-Yong N, Zhang QL. Lyapunov and non-Lyapunov Stability of Linear Discrete Time Delay Systems. Facta Universitatis, Series: Mechanical Engineering. 2002: 1(9): 1147-1160

[53] Dzieliński A, Sierociuk D. Observers for discrete fractional order systems, Proceedings of the 2nd IFAC Workshop on Fractional Differentiation and its Applications (FDA'06); 2006 Jul 19-21; Porto, Portugal.

[54] Dzieliński A, Sierociuk D. Stability of discrete fractional-order state-space systems, Proceedings of the 2nd IFAC Workshop on Fractional Differentiation and its Applications (FDA'06): 524-529; 2006 Jul 19-21; Porto, Portugal.

[55] Lall S, Beck C. Error-bounds for balanced model-reduction of linear time-varying systems, IEEE Trans Automat Contr. 2003; 48(6):946-956

[56] Dullerud G, Lall S. A new approach for analysis and synthesis of time-varying systems, IEEE Trans Autom Contr. 1999; 44: 1486-1497 
[57] Garcia G, Messaoud H, Maraoui S. Practical stabilization of linear time-varying systems. Sixth International Conference on Sciences and Techniques of Automatic control; 2005 Dec 19-21, Sousse, Tunisia.

[58] Doyle JC. Lectures notes in advances in multivariable control, ONR/Honeywell Workshop, Minneapolis, 1984.

[59] Curtain R, Morris K. Transfer functions of distributed parameter systems: A tutorial. Automatica. 2009; 45: 1101-1116

[60] Iglesias PA (The Johns Hopkins University, Department of Electrical and Computer Engineering, Baltimore, MD 21218). On the stabilization of discrete-time linear time-varying systems. pi@ruth.ece.jhu.edu. Technical report JHU/ECE-94/08; 1994 May 8. 